\title{
The Effect of Coconut Shell Powder as Functional Filler in Polypropylene during Compounding and Subsequent Molding
}

\author{
Matt Kirby ${ }^{\mathrm{a}}$, Benjamin Lewis ${ }^{\mathrm{a}}$, Benjamin Peterson ${ }^{\mathrm{a}}$, Juliana Anggono ${ }^{\mathrm{b}}$, Walter Bradley ${ }^{\mathrm{a}^{*}}$ \\ ${ }^{a}$ Baylor University, 1301 S University Parks Dr, Waco, TX 76706, United States \\ bPetra Christian University, Jalan Siwalankerto 121-131 Surabaya 60236, Indonesia
}

\begin{abstract}
Fine powder produced from coconut shells (CSP) has been found to be excellent functional filler in polypropylene, significantly increasing flexural and tensile moduli, slightly increasing tensile strength, and good retention of Izod impact strength at weight percentages less than $20 \%$. The research presented in this paper explores the temperature processing window for coconut shell powder filled polypropylene and the change in the rheological behavior of the polypropylene due to the addition of CSP as functional filler in this processing temperature window. The reason for the simultaneous increase in flexural modulus, tensile strength, and good retention of Izod impact behavior is also investigated
\end{abstract}

Key words: Functional filler, impact strength, natural filler reinforcement, polymeric composites, rheological behavior.

\section{Introduction}

There is a growing interest in making polymeric composite materials that are more sustainably by using functional fillers that are renewable. Coconut shell powder (CSP) produced from discarded coconut shells in the production of coconut oil has been found to be a very effective functional filler in polypropylene because CSP is a lignin rich biomass that is four times harder than hard maple and other hardwoods [1]. The addition of $10 \mathrm{wt} \%$ to $20 \mathrm{wt} \%$ CSP to polypropylene (PP) can increase the flexural modulus from $40 \%$ to $70 \%$, the tensile strength from $30 \%$ to $50 \%$ with good retention of Izod impact strength [2].

These exciting results have stimulated a keen interest to know how the incorporation of CSP into PP will affect its processing. How will the upper and lower bound temperatures which define the processing window for compounding and for injection molding or extruding change when CSP is used as functional filler in PP. An equally important question is how is the rheological behavior of the PP is altered with the addition of various amounts of coconut shell powder? A third significant question is how can the coconut shell powder increase the strength and stiffness of polypropylene with good retention of its Izod impact strength, which is most unusual?

${ }^{*}$ Corresponding author: Walter Bradley@baylor.edu 


\section{Literature survey}

\subsection{Maximum allowable processing temperature}

The upper bound for the thermal processing window is usually determined by thermal degradation, which occurs through thermally induced breaking of covalent bonds. While it is normally possible to process most thermoplastics at temperatures well below the temperature at which they thermally degrade, most natural filler will have somewhat lower thermal degradation temperature than $\mathrm{PP}$, which may require the thermoplastic to be processed at a lower temperature than would normally be used. Most lingo-cellulosic biomass contains three microstructural constituents: lignin, cellulose, and hemi-cellulose. Thermal degradation begins for such biomass around $200{ }^{\circ} \mathrm{C}$ [3]. Polypropylene is usually processed between $180^{\circ} \mathrm{C}$ and $230^{\circ} \mathrm{C}$. Therefore, the specific degradation temperature and behavior of each potential natural filler must be fully understood before it can be considered as functional filler for PP.

Since thermal degradation is a thermally activated breaking of chemical bonds, it proceeds at a rate that is defined by the Arrhenius relationship for thermally activated processes in nature; namely, $(\sim \exp [-Q / k T])$ [2]. However, the total amount of degradation depends on the time spent at that temperature. For example, natural fillers that thermally degrade by $5 \mathrm{wt} \%$ at $200{ }^{\circ} \mathrm{C}$ in $60 \mathrm{~min}$ might thermally degrade by $5 \mathrm{wt} \%$ at $230{ }^{\circ} \mathrm{C}$ in $3 \mathrm{~min}$. Thus, the selection of the "maximum permissible temperature" for the processing window will depend on the anticipated time that the polymer and functional filler will be in the extruder or injection molding machine barrel. The effect of time on thermal degradation at different temperatures has been well documented for wood fillers and other lingo-cellulosic fillers such as hemp and flax $[4,5]$. The thermal degradation of coconut shell has also been studied extensively by Macedo et al. [6]. However, these studies were done using a thermo-gravimetric analyzer (TGA) using only ramp heating, making it difficult to determine the accumulated degradation as a function of time at various processing temperatures. Furthermore, they used pieces of coconut shell whereas in this work, coconut shell powder were used with a diameter of $\sim 150 \mu \mathrm{m}$ and a much greater surface area. Finally, to give guidance to compounders and injection molders, this research had an interest in the rate of degradation for coconut shell powder imbedded in PP, which would delay for some time the onset of thermal degradation just due to the thermal inertia associated with heating up PP pellets with CSP already compounded into them.

\subsection{Minimum allowable processing temperature}

The lower bound of the processing window of a semi-crystalline thermoplastic polymer like PP is defined by the onset of crystallization. Like thermal degradation, the rate of crystallization is a function of temperature, but for a somewhat more complex process than thermal degradation, involving as it does nucleation of small crystals and their subsequent growth [7]. As the temperature is lowered below the equilibrium melting temperature (which is called super cooling), the time to nucleate and grow spherulites becomes progressively shorter. However, a very rapid quench can avoid the formation of spherulites altogether. The processing temperature in the injection molding of larger, complex parts needs to be done at a temperature sufficiently above the temperature at which 
crystallization begins to allow the mold to be completely filled before cooling leads to crystallization, which can cause cold shuts.

While the lower bound temperature for the processing window is well known for various PP polymers, it is possible that this window might change for PP with CSP. The CSP may provide heterogeneous nucleation sites that would make the nucleation of spherulites much easier, and therefore allow crystallization to begin at a higher temperature (at a smaller degree of super cooling). Thus, the processing window with CSP is definitely lower on the high temperature side and higher on the low temperature side, resulting in a smaller processing temperature window.

The unusual combination of CSP in PP enhancing tensile strength, flexural modulus and having good retention of Izod impact strength may be the result of heterogeneous nucleation of spherulites on the CSP particles, giving a much finer spherulitic structure, which would in turn give more tie molecules, resulting in greater tensile strength and greater Izod impact strength [7]. A higher $\mathrm{wt} \%$ of crystallinity might also result from heterogeneous nucleation, giving a higher flexural modulus.

\subsection{Rheological behavior of CSP filler PP in the temperature window for processing}

The addition of fillers to PP is known to increase its viscosity [8]. The extent of this increases is largely based on several characteristics of the filler such as: materials type, size, aspect ratio, surface roughness and $\mathrm{wt} \%$ loading [8]. It is essential that the change in viscosity with wt\% loading of CSP in PP be determined for a wide range of shear rates over the range of temperatures in the processing window. This will make it possible to understand the tradeoffs between enhancement of the mechanical properties of PP with CSP filler and the increase in degree of difficulty in molding with this filled polymer. The impact of CSP on the rheological behavior of PP has not previously been studied.

\section{Experimental plans and procedures}

The four research questions that will be addressed deal with how the use of CSP as functional filler in PP affects:

i. The maximum allowable processing temperature in the processing temperature window;

ii. The minimum allowable processing temperature in the processing temperature window;

iii. The rheological behavior of the polymer in the temperature processing window;

iv. The PP crystallinity and morphology to give the filled PP a uniquely attractive combination of tensile strength, flexural modulus, and Izod impact strength.

\subsection{Materials}

The polypropylene (PP) used in this investigation is a polypropylene copolymer that is used in the automotive industry for injection molded parts. The copolymer is primarily polypropylene with some polyethylene to enhance low temperature impact properties. The

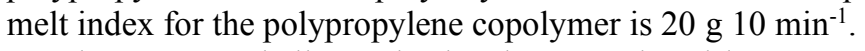

The coconut shell powder has been produced by processing coconut shell into a fine powder with a maximum particle size of $150 \mu \mathrm{m}$ and a median particle size of $65 \mu \mathrm{m}$. To provide good interfacial bonding between the CSP and PP, $2 \%$ maleic anhydride polypropylene (MAPP) was added as a compatibilizer. 


\subsection{Maximum allowable processing temperature}

The maximum allowable processing temperature is determined by the thermal degradation of the CSP functional filler in the PP. The thermal degradation was studied using mass loss as a function of time and temperature to track the degradation. These measurements of mass loss were made on a Thermal Analysis (TA) Q50 Thermogravimetric Analyzer. Coconut shell powder and coconut shell powder compounded into PP were both studied. Powder sample masses were $(13.5 \pm 2) \mathrm{mg}$ and pellet sample masses were $(23.5 \pm 2) \mathrm{mg}$ to minimize the variability due to thermal transients in the samples. Two temperature-time histories were used to map out the thermal degradation as a function of temperature and time-at-temperature. First, a constant heating ramp of $2{ }^{\circ} \mathrm{C} \min ^{-1}$ was performed; and second, a rapid heating to a fixed temperature followed by an isothermal hold was conducted.

\subsection{Minimum allowable processing temperature and effect of CSP on wt\% crystallinity}

The minimum allowable processing temperature in PP is determined by the onset of crystallization. The crystallization that occurs during cooling of PP can be studied by measuring the latent heat of crystallization that is released as the PP has a phase change from amorphous to crystalline PP. The crystallization kinetics of PP with and without CSP functional filler was studied by measuring the release of the latent heat of crystallization using a Thermal Analysis (TA) Q20 Differential Scanning Calorimeter. The measurement of heat released as a function of temperature and time allow the temperature at which crystallization begins and the temperature at which the crystallization process has ceased to be determined. The total energy released per gram of material in the DSC allows the wt $\%$ crystallinity to be determined as well. Polypropylene with $0 \mathrm{wt} \%, 10 \mathrm{wt} \%$ and $20 \mathrm{wt} \%$ CSP was studied. The sample masses were $12 \pm 3 \mathrm{mg}$ to minimize thermal transients in the samples.

\subsection{Effect of CSP on rheological behavior of PP in thermal processing window}

The primary objective of the rheological research is to determine the effect of CSP on the viscosity of PP. Two secondary objectives are to determine the additional effects of moisture content and degradation of the CSP on the viscosity of the CSP filled PP.

A Tinius Olsen MP600 Melt-Plastometer (melt flow indexer) was used to measure apparent viscosity versus shear rate from $100\left(1 \mathrm{~s}^{-1}\right)$ to $1000\left(1 \mathrm{~s}^{-1}\right)$. Calculations proposed by Shenoy et al [9] for obtaining rheological data from a melt flow indexer were used. Neat PP pellets and PP pellets compounded with $10 \mathrm{wt} \%, 20 \mathrm{wt} \%$, and $30 \mathrm{wt} \%$ CSP powder were prepared using CSP that had been dried in a furnace at $160{ }^{\circ} \mathrm{C}$ for $2 \mathrm{~h}$, reducing the moisture content from $6.7 \mathrm{wt} \%$ to $1 \mathrm{wt} \%$ until $2 \mathrm{wt} \%$. The resultant pellets of PP with CSP filler were subsequently dried briefly at $90{ }^{\circ} \mathrm{C}$ to remove surface moisture just before loading them into the barrel of the Melt-Plastometer. The melt time in the barrel for the pellets was approximately three minutes to minimize thermal degradation of the CSP in the pellets. Fixed loads were applied to the piston of the melt flow indexer and the volumetric flow-rate was measured. From this data, the apparent viscosity vs. shear rate was determined for loadings of $10 \mathrm{wt} \%, 20 \mathrm{wt} \%$, and $30 \mathrm{wt} \%$ CSP.

The effect of moisture in the CSP at the time of compounding on the viscosity versus shear rate was also studied. CSP with $6.7 \mathrm{wt} \%$ moisture was compounded into PP. The 
resulting pellets were tested as described in the preceding paragraph and compared to results on PP with CSP that was dried to $1 \mathrm{wt} \%$ to $2 \mathrm{wt} \%$ moisture before compounding.

To explore the effect of thermal degradation on the viscosity versus shear rate, the rheological measurements described above were repeated on PP with $30 \mathrm{wt} \%$ CSP using a dwell time in the barrel of the melt flow indexer of $3 \mathrm{~min}$ and $10 \mathrm{~min}$ to allow more time for degradation. The results for these two different dwell times in the barrel were compared to indicate what if any effect thermal degradation might have on the rheological properties of PP filled with $30 \mathrm{wt} \%$ CSP

\section{Results and discussion}

\subsection{Maximum allowable processing temperature}

The most important limitation in the use of natural fillers in thermoplastics is the thermal degradation temperature. If this temperature is below the usual processing temperature for a given plastic, it cannot be used as functional filler in that polymer. The TGA results for coconut shell powder (CSP) are presented in Figure 1 along with results for powders (sometimes called flour) made from rice hulls, walnuts shells and pine wood.

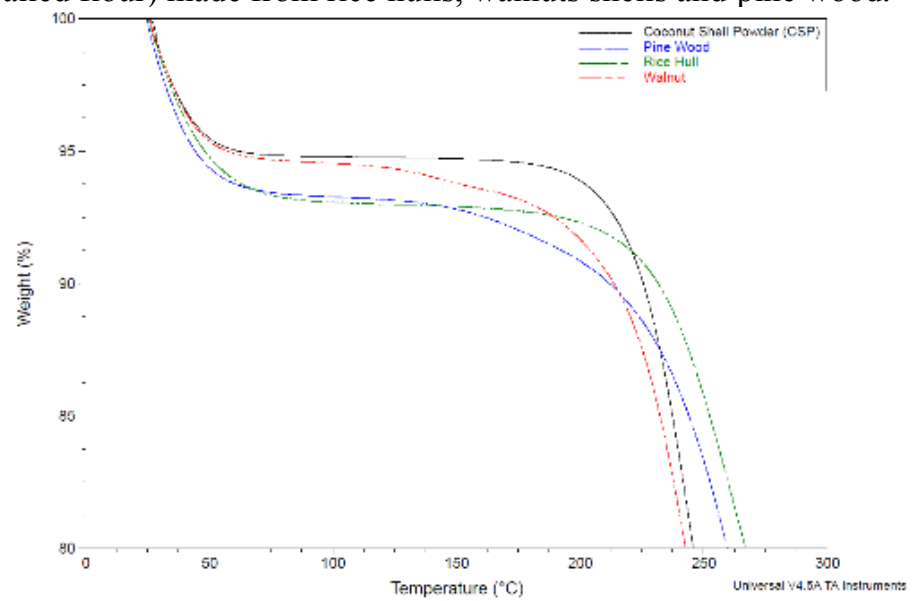

Fig. 1. Thermal degradation of natural fillers $\left(2^{\circ} \mathrm{C} \mathrm{min}^{-1}\right)$

The initial drop in weight is due to the removal of water from these natural fillers. This was $6.7 \mathrm{wt} \%$ in CSP. The subsequent deviation from the plateau is due to thermal degradation, which converts some of the biomass into volatiles, with a resultant loss in mass. The powders made from walnuts and pine wood (and also wheat straw and maple which are not shown) begin to thermally degrade at temperature of $160{ }^{\circ} \mathrm{C}$ or less. The melt temperature for polypropylene and copolymers of polypropylene is approximately $170{ }^{\circ} \mathrm{C}$, with processing temperature somewhat higher. Thus, many natural fillers will degrade, smoke and smell when processed in polypropylene. Coconut shell powder and rice hulls have less than $1 \%$ wt loss at $200{ }^{\circ} \mathrm{C}$. This higher degradation temperature is why CSP can be compounded into PP using an extruder without any significant thermal degradation.

Most plant biomass is comprised of hemicelluloses, cellulose, and lignin with lignin being the most resistant to thermal degradation. The thermal degradation temperature of the biomass will depend on the relative mass fraction of each of these constituents and their arrangement in the microstructure of the biomass. Coconut shell has one of the highest lignin contents of any plant biomass, and it is this high lignin content that makes it resistant to thermal degradation and resistant to microbial attack as well [10]. 
The temperature requirements for injection molding are higher than for compounding, and especially so if one is making larger and/or more complex parts. Fortunately, the resistance to thermal degradation of the CSP is somewhat greater when it is encapsulated in pellets of PP, as there is a thermal lag associated with the heat transfer through the PP. The thermal degradation of CSP filled PP is presented in Figure 2. TGA measurements with a ramp of $2{ }^{\circ} \mathrm{C} \mathrm{min}^{-1}$ are presented for neat PP, and PP with 10 mass $\% \mathrm{CSP}, 20$ mass $\% \mathrm{CSP}$, and 30 mass $\%$ CSP. The results from Figure 1 for CSP that has not been encapsulated are included for comparison.

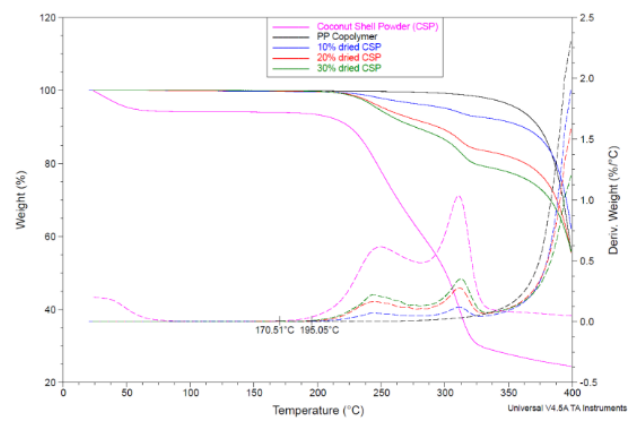

Fig. 2. Thermal degradation of neat PP, and PP with 10 mass $\%$ CSP, 20 mass $\%$ CSP, and 30 mass $\%$ CSP. CSP that is not encapsulated is also included for comparison.

The derivative of weight loss versus temperature is also included to allow one to see more accurately the onset of degradation. The comparison of the CSP and the PP curves demonstrate the much greater thermal stability of the PP compared to the CSP. The three curves in-between show the thermal degradation of the CSP encapsulated in the PP. The delayed onset and the slower rate of thermal degradation of the CSP in the PP is evident and due to thermal lag associated with heat transfer through the PP, which has a low thermal conductivity. This increases the allowable temperature for injection molding over what is permissible in compounding by about $25^{\circ} \mathrm{C}$. It is also worth noting that the CSP was dried to $1 \mathrm{wt} \%$ to $2 \mathrm{wt} \%$ moisture before being compounded into the PP, which is why there is almost no weight loss due to moisture for the PP pellets, with or without CSP.

The rate of degradation of CSP encapsulated in PP at $210{ }^{\circ} \mathrm{C}$ was determined by isothermal tests performed using the TGA. The purpose of these tests was to quantify the level of degradation that might take place during the dwell time in the barrel of an extruder or injection molding machine. The results are presented in Figure 3.

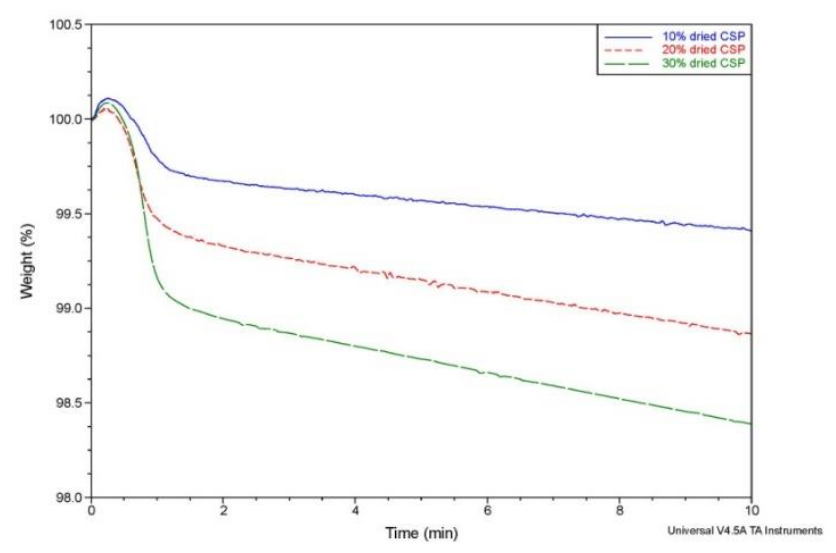


Fig. 3. Thermal degradation of CSP filled PP at $210^{\circ} \mathrm{C}$

The initial drop of $\sim 1 \mathrm{wt} \%$ in the first minute at $210{ }^{\circ} \mathrm{C}$ is due to loss of the residual $1 \%$ moisture that is still present in the coconut shell powder after drying. The slopes of the three curves indicate a rate of weight loss of the composite of $0.025 \mathrm{wt} \% \mathrm{~min}^{-1}, 0.050 \mathrm{wt} \%$ $\mathrm{min}^{-1}$ and $0.075 \mathrm{wt} \% \mathrm{~min}^{-1}$ of composite for $10 \mathrm{wt} \%, 20 \mathrm{wt} \%$ and $30 \mathrm{wt} \%$ respectively of CSP filling in the PP. Assuming all of the degradation takes place in the CSP, which is justified by the results in Figure 2, all three curves correspond to a weight loss of CSP of $0.25 \mathrm{wt}^{\%} \mathrm{~min}^{-1}$ at $210{ }^{\circ} \mathrm{C}$. This remarkably low rate of CSP degradation at $210{ }^{\circ} \mathrm{C}$ is consistent with Figure 2 and with the experience of a number of commercial compounders and injection molders who have been pleasantly surprised at how easily coconut shell powder can be compounded into PP and subsequently injection molded without the smoking, smelling and associated degradation that is often found with other natural fillers.

\subsection{Minimum allowable processing temperature and effect of CSP on wt\% crystallinity}

The differential scanning calorimeter measurements were designed to determine if coconut shell powder (CSP) not only served directly as a stiffener of the PP but also indirectly as a stiffener by increasing the $\mathrm{wt} \%$ of crystallinity in PP by acting as a nucleating agent for crystallization. The results are presented in Figures 4 to Figure 6 . The melting curve corresponds to second heating so that the data corresponds to the first cooling and crystallization in the PP. The rate of cooling and heating was $10{ }^{\circ} \mathrm{C} \mathrm{min}-1$.

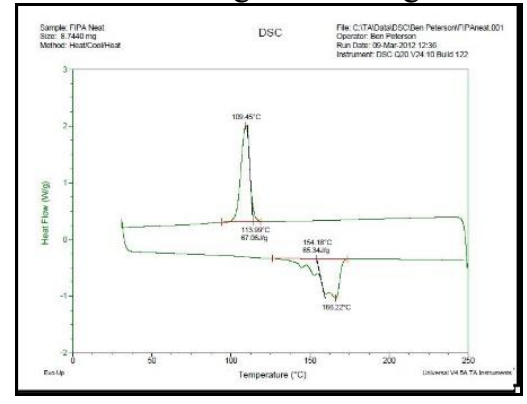

Fig. 4. DSC results for neat polypropylene co-polymer.

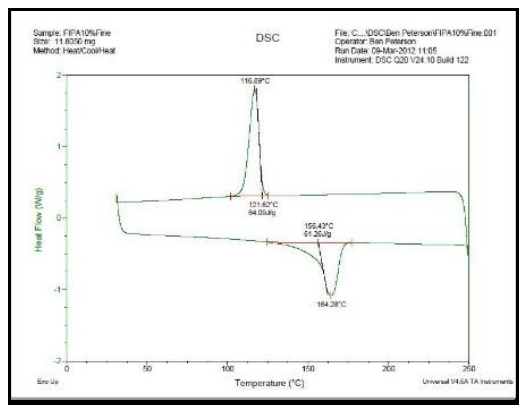

Fig. 5. DSC results for polypropylene co-polymer with $10 \mathrm{wt} \%$ CSP filler. 


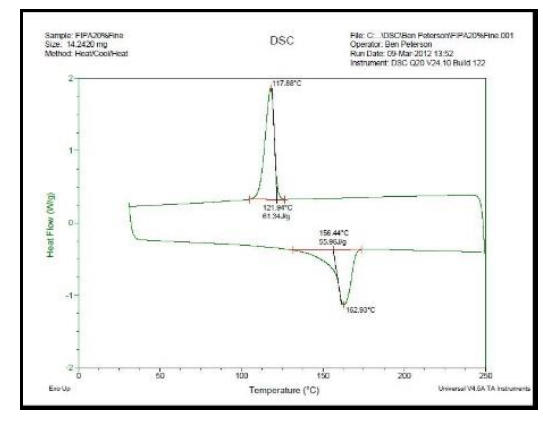

Fig. 6. DSC results for polypropylene co-polymer with $20 \mathrm{wt} \% \mathrm{CSP}$.

The two most interesting observations of the DSC results in Figures 4 to Figure 6 are (i) the changes in the temperature at which crystallization begins for the neat PP copolymer compared to the PP copolymer with CSP filler and (ii) the total energy released during crystallization, which corresponds to the wt $\%$ of crystallinity in the PP copolymer.

If one considers the onset of melting as $\mathrm{T}_{\mathrm{m}}$ and the onset of crystallization as $\mathrm{T}_{\mathrm{c}}$, then the degree of super cooling $\Delta \mathrm{T}_{\mathrm{s}}$ required to nucleate crystallization at the cooling rate imposed by the DSC is

$$
\Delta e T_{s}=T_{m}-T_{c}
$$

For the neat PP copolymer, the super cooling $\Delta \mathrm{T}_{\mathrm{s}}$ required to nucleate spherulites is $13.5^{\circ} \mathrm{C}$. For the PP copolymer with $10 \mathrm{wt} \% \mathrm{CSP}$ filler and $20 \mathrm{wt} \% \mathrm{CSP}$ filler; the observed super cooling is only $6.1{ }^{\circ} \mathrm{C}$ and $5.8{ }^{\circ} \mathrm{C}$ respectively. This clearly suggests that the coconut shell powder is functioning as a nucleating agent, facilitating heterogeneous nucleation. This would also suggest that the spherulites size should be smaller in filled PP copolymer, which would provide more tie molecules between spherulites and account for the excellent Izod impact strength with additions of $10 \mathrm{wt} \%$ CSP or $20 \mathrm{wt} \%$ CSP.

The area under the crystallization curves can be used to determine the $\mathrm{J} \mathrm{g}^{-1}$ of heat released during crystallization. After correcting for the wt\% of CSP, the measured heat released during crystallization per gram of PP copolymer in the three polymeric composites are as follows: neat-67.1 $\mathrm{J} \mathrm{g}^{-1} ; 10 \mathrm{wt} \%$ CSP-71.2 $\mathrm{J} \mathrm{g}^{-1}$; and $20 \mathrm{wt} \%$ CSP-76.7 $\mathrm{J} \mathrm{g}^{-1}$.

The measure heat released per gram of PP copolymer can then be used to estimate the degree of crystallization if the heat of fusion for crystallization is known. Unfortunately, the heat of fusion is not known precisely for the PP copolymer used in this investigation. The heat of fusion can vary from $163 \mathrm{~J} \mathrm{~g}^{-1}$ to $118 \mathrm{~J} \mathrm{~g}^{-1}$ as the ethylene in the PP copolymer increases from $0 \mathrm{wt} \%$ to $18 \mathrm{wt} \%$ [11]. The neat PP copolymer would have a degree of crystalline of between $41 \mathrm{wt} \%$ and $57 \mathrm{wt} \%$ depending on the $\mathrm{wt} \%$ polyethylene in the proprietary copolymer. The $10 \mathrm{wt} \% \mathrm{CSP}$ system would have a crystallinity of between $44 \mathrm{wt} \%$ and $60 \mathrm{wt} \%$ and the $20 \mathrm{wt} \%$ SCP system would have a crystallinity between $47 \mathrm{wt} \%$ and $65 \mathrm{wt} \%$. As the wt $\%$ CSP increases, the wt $\%$ of crystallinity increases by about $15 \%$ (from $41 \mathrm{wt} \%$ to $47 \mathrm{wt} \%$, for example). The increase in degree of crystallization provides additional evidence to support the hypothesis that the CSP serves as a nucleating agent in the PP copolymer.

While this increase in crystallinity accounts for some of the observed $70 \%$ increase in flexural modulus, it cannot account the whole increase that results when CSP is used as a functional filler in PP copolymers. Thus, the CSP must also be contributing directly to the increase in stiffness by acting as a hard second phase particle in the PP copolymer beyond the benefits it provides as a nucleating agent. 


\subsection{Effect of CSP on rheological behavior of PP in thermal processing window}

The effect of CSP on the apparent viscosity is seen in Figure 7, characterizing the change of viscosity for four different loadings of CSP.

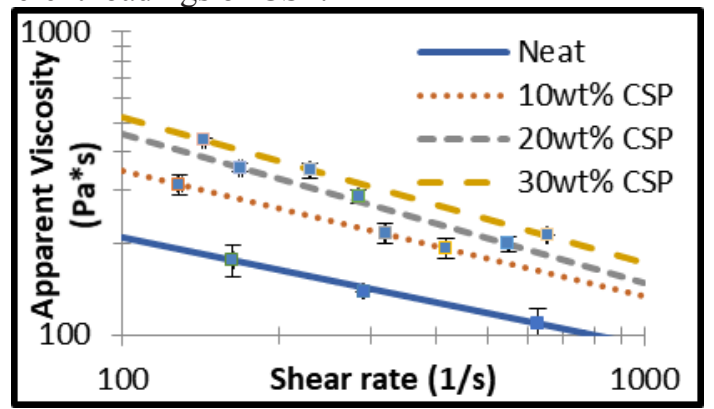

Fig. 7. Apparent viscosity versus shear rate for neat PP copolymer with and without CSP at $210{ }^{\circ} \mathrm{C}$

The results indicate that the viscosity of the filled polypropylene increases with increasing percent loading of CSP. However, the observed increase is quite small compared to other fillers. A second anomaly in the influence of CSP on viscosity is that increasing increments of CSP have a reduced impact on the viscosity, as seen in Figure 8. Shenoy [1] has noted in this regard, "With increasing concentration of the filler, the inter-particle interactions increase weakly at first and then rather strongly as the concentration becomes higher and higher." Therefore, it could be expected that viscosity is more sensitive to filler loading changes at higher loadings, which is clearly not the case for CSP filler in this PP copolymer, as seen in Figure 8.

These small relative viscosity values and decreasing viscosity sensitivity at higher filler loadings allow the CSP composite to process easily without requiring an excess of additional energy in the form of heat or injection pressure. The reason for this decreasing incremental impact of CSP on the viscosity of PP copolymer seen in Figure 8 could be organic nature of CSP and/or the shape of the CSP particles, as seen in Figure 9. The CSP particles are seen to be globular rather than fibrous, and this would account in part for their relatively small impact on the viscosity of the PP copolymer, as seen in Figure 7.

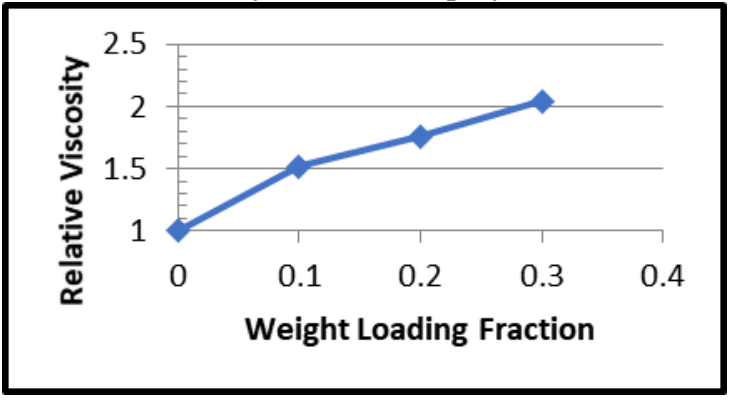

Fig. 8. Relative viscosity vs. weight loading fraction at $210^{\circ} \mathrm{C}$ at the shear rate of $500\left(1 \mathrm{~s}^{-1}\right)$ 


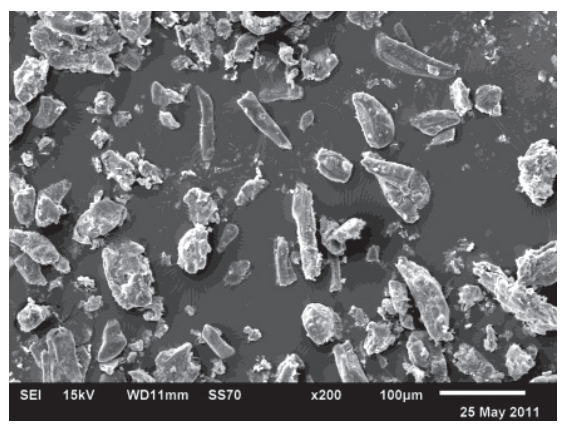

Figure 9. SEM photograph of CSP.

It was hypothesized that moisture and thermal degradation of the CSP could increase molecular mobility, and therefore, decrease viscosity. The amount of both moisture in the CSP and thermal degradation of the CSP would increase with an increase in weight percent loading in a composite, which might account for the observations in Figure 8 . The viscosity for PP copolymer with $20 \mathrm{wt} \%$ CSP with $2 \mathrm{wt} \%$ moisture and with $6.7 \mathrm{wt} \%$ moisture is compared in Figure 10. The effect of moisture is seen to be minimal.

The results in Figure 11 can be used to infer whether thermal degradation can have an impact on the viscosity of CSP filler PP copolymer. The results in Figure 3 indicate that 3 min dwell time in the barrel of an injection molding machine at $210{ }^{\circ} \mathrm{C}$ would thermally degrade $0.75 \mathrm{wt} \%$ of CSP $(0.225 \mathrm{wt} \%$ of composite $)$ while $2.5 \mathrm{wt} \%$ of CSP $(0.75 \mathrm{wt} \%$ of composite) would degrade in $10 \mathrm{~min}$. The results in Figure 11 indicate that increasing thermal degradation does reduce viscosity, though not dramatically.

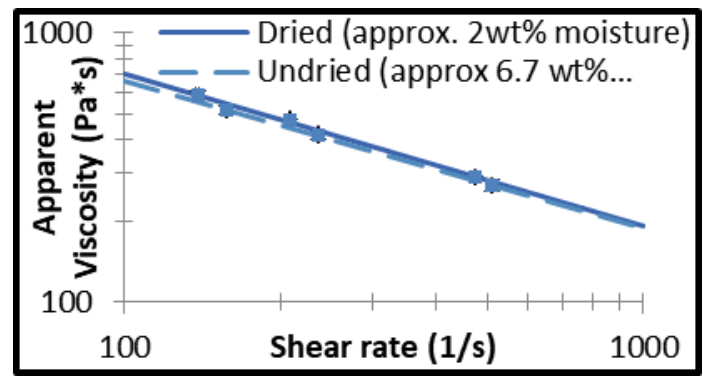

Fig. 10. Effect of moisture on rheology with $20 \%$ CSP loading at $180^{\circ} \mathrm{C}$

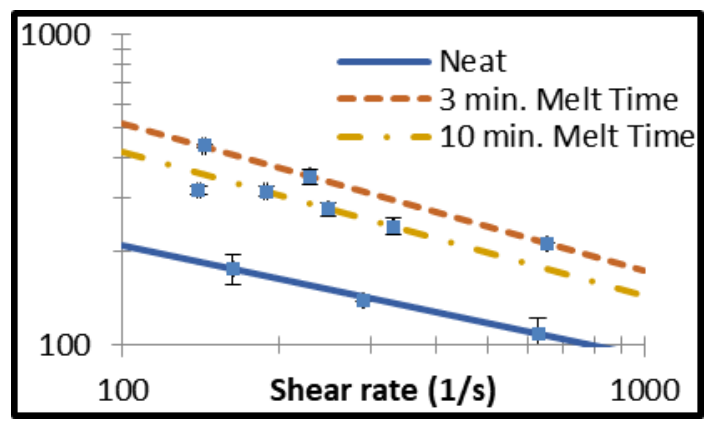

Fig. 11. Effect on viscosity of thermal degradation of $30 \mathrm{wt} \% \mathrm{CSP}$ filled PP copolymer at $210{ }^{\circ} \mathrm{C}$. 


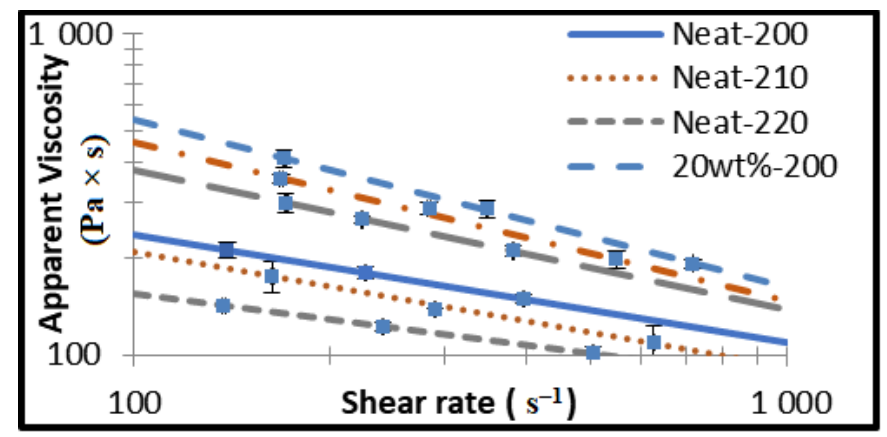

Fig. 12. The apparent viscosity vs shear rate for neat PP copolymer and filled with $20 \mathrm{wt} \% \mathrm{CSP}$.

Finally, the viscosity as a function of shear rate for neat and CSP filled (20 wt $\%$ ) PP copolymer at three different temperatures is presented in Figure 12. The behavior at each temperature shows only a modest $\sim 2$ times increase in viscosity due to the addition of the $20 \mathrm{wt} \%$ CSP filler.

\section{Summary}

The processing characteristics of coconut shell powder used as function filler in polypropylene are very attractive. The following specific characteristics were established in this study.

i. Coconut shell powder is high in lignin compared to most natural fillers, which gives it a higher thermal degradation temperature than most natural fillers.

ii. Coconut shell powder filled polypropylene copolymer used in this study (and presumably in most polypropylenes) should compound and injection mold without thermal degradation issues.

iii. Coconut shell powder used as a function filler in polypropylene copolymer serves as a spherulite nucleating agent, increasing the temperature at which crystalline begins (by reducing the required super cooling need for nucleation) and increasing the $w t \%$ crystallization. It presumably decreases the spherulites size, which would account for the good retention of Izod impact properties, though this was not directly confirmed by microscopy.

iv. Coconut shell powder is an excellent function filler due to its relatively low viscosities in the shear-thinning region, even at higher loading levels. This allows the CSP composite to be processed easily. Furthermore, very small amounts of thermal degradation of the CSP give a modest decrease in viscosity, behaving like an internal processing aid in the polypropylene.

$\mathrm{v}$. The excellent combination of properties of coconut shell powder as a functional filler in polypropylene copolymer explain why CSP has been give "superior marks" by a wide variety of compounders and injection molders in industry who have used it with polypropylene or polypropylene copolymers.

Coconut shell powder is a very attractive functional filler for polypropylene because it provides a unique combination of increased flexural modulus and Izod impact strength with superior process-ability. 


\section{Acknowledgments}

This work was done under the auspices of Natural Composites Inc. who also provided financial support for the project and supplied the polypropylene copolymer, coconut shell powder, and MAPP. The SEM picture in Figure 9 was selected from a larger group of SEM pictures taken by Sarah Stair. Updating references was assisted by Benny Putra Utomo

\section{References}

1. E.G. Teipel, S. Conroy, M. Kirby, W. Bradley. Natural functional fillers from agricultural waste to reinforce polymeric composites: coconut shell powder technology Paper presented in SPE Polyolefin Conference, (Houston, Texas, 2012). https://www.researchgate.net/publication/286214575 Natural functional fillers from agricultural_waste to reinforce polymeric_composites_Coconut shell_powder_techn ology

2. I.M. Ward, J. Sweeney. An introduction to the mechanical properties of solid polymers. England: John Wiley \& Sons Inc. (2004). pp. 104-107. https://www.wiley.com/en-

$\underline{\mathrm{id} / \text { An }+ \text { Introduction }+ \text { to }+ \text { the }+ \text { Mechanical }+ \text { Properties }+ \text { of }+ \text { Solid }+ \text { Polymers, },+2 \text { nd }+ \text { Edition }}$ -p-9780471496267

3. H. Yang, R. Yan, H. Chen, D.H. Lee, C. Zheng. Fuel, 86,12-13:1781-1788 (2007). https://www.sciencedirect.com/science/article/pii/S001623610600490X

4. D.L. Motoc, S.F. Bou, A.P. Pop. MATEC Web of Conferences, 184,11:01016 (2018). https://www.researchgate.net/publication/326712780 Thermal degradation and stabil ity of wood particle composites deployed as decorative components

5. N. Stevulova, A. Estokova, J. Cigasova, I. Schwarzova, F. Kacik, A. Geffert. Journal of Thermal Analysis and Calorimetry, 128,3:1649-1660 (2016). https://link.springer.com/article/10.1007/s10973-016-6044-z

6. J.S. Macedo, L. Otubo, O.P. Ferreira, I.F. Gimenez, I.O. Mazali, L.S. Barreto. Microporous and Mesoporous Materials, 107,3:276-285(2008). https://www.sciencedirect.com/science/article/abs/pii/S138718110700176X

7. G.W. Ehrenstein. Polymeric composites. Munich: Hanser (2001), pp. 63-92.

8. S.C. Koay, M.Y. Chan, M.M. Pang, K.Y. Tshai. Advances in Polymer Technology, 37,6: 2246-2252 (2018). https://onlinelibrary.wiley.com/doi/full/10.1002/adv.21883

9. A.V. Shenoy, D.R. Saini. Thermoplastic melt rheology and processing. New York: CRC Press (1996). pp. 115-172.

https://books.google.co.id/books?id=lksD1Mq5twIC\&dq=thermoplastic + melt + rheolog $\mathrm{y}+$ and + processing \&hl $=$ en \&sa $=$ X\&ved $=0$ ahUKEwiUiyEhuThAhWz7HMBHfooDlcQ6AEIKjAA

10. S. Conroy. The efficacy of using coconut shell powder as a filler/reinforcement in thermoplastic composites. Master's Thesis, Baylor University, Waco, Texas (2011).

11. J.T. Uan-Zo-li. Morphology, crystallization and melting behavior of propyleneethylene statistical copolymer. [Dissertation Ph.D.]. Virginia Polytechnic Institute and State University, Blacksburgm Virginia (2005).

https://vtechworks.lib.vt.edu/handle/10919/29143 
